\title{
Organ Specific Cancers - Recent Advances in Diagnosis and Treatment
}

\author{
Shalini G ${ }^{1 *}$, Remya RS ${ }^{2}$, Gayathri G ${ }^{3}$, Vishalakshi V ${ }^{4}$ and Phaneendra M $^{5}$ \\ ${ }^{1}$ Department of Biochemistry, Andhra University, Andhra Pradesh, India \\ ${ }^{2}$ Department of Biotechnology, Kerala University, India \\ ${ }^{3}$ Department of Microbiology, Yogi Vemana University, Andhra Pradesh, India \\ ${ }^{4}$ Department of Microbiology, Osmania University, Andhra Pradesh, India \\ ${ }^{5}$ Department of Biotechnology, GITAM University, Andhra Pradesh, India
}

\begin{abstract}
Cancer is one of the leading causes of death all over the world. Organ Specific Cancers are cancers named baseing on the location of cancer in the body organ. Its incidence is showing an increasing trend in various parts of the earth and has been a significant public health problem despite advances in the understanding of the molecular and cellular events that underlie. This review aims to update the current related information about organ specific cancers and provide a further understanding about their diagnosis and treatment.
\end{abstract}

Keywords: Organ specific cancers; Cancer treatment; Brain cancer; Lung cancer

\section{Introduction}

Cancer is the second leading cause of death in the world after cardiovascular diseases. Half of men and one third of women in the United States will develop cancer during their lifetimes. There are many old theories about cancers like humoral theory, lymph theory, blastema theory, chronic irritation theory, trauma theory, parasite theory. By the middle of the 20th century, scientists began solving the complex problems of chemistry and biology behind cancer. During the 1970s, scientists discovered 2 important families of genes i.e. oncogenes and tumor suppressor genes. The continuing research is discovering new carcinogens, explaining how they cause cancer, providing insight into ways to prevent and treat [1].

\section{Types of Cancers}

There are different types of cancers based on location of cancer in the body organ. For example: Brain cancer, Head and Neck Cancer, Oral cancer, Lung cancer, Liver cancer, Breast cancer, Prostate cancer, Gastric cancer, Pancreatic cancer, Kidney (Renal Cell) cancer, Leukemia etc. These types of cancers come under the category of Organ Specific Cancers. Let us discuss in detail about these organ specific cancers in this review article.

\section{Brain cancer}

One of the most devastating CNS pathologies is brain cancer. Cancers of the brain are the consequence of abnormal growths of cells in the brain. Tumors in the brain can be malignant or benign, and can occur at any age. Only malignant tumors are cancerous. The visibility of signs and symptoms of brain tumors mainly depends on two factors: tumor size (volume) and tumor location. The moment that symptoms will become apparent, either to the person or people around him (symptom onset) is an important milestone in the course of the diagnosis and treatment of the tumor. Early and accurate diagnosis of small metastatic brain tumors may affect outcomes and treatment strategies. For this reason, 3-dimensional (3D) thin-section imaging is preferred [2]. Tumors recruit normal CNS stem and progenitor cells to the tumor mass leading to the possibility of a heterogeneous and polyclonal cell population. It is likely that a complete description of the role of stem cells in brain tumors will be more complex than our current models [3].
Microwave exposure from the use of cellular telephones has been discussed in recent years as a potential risk factor for brain tumors. With regard to different tumor types, the highest risk was for acoustic neurinoma (OR 3.5, 95\% CI 1.8-6.8) among analogue cellular telephone users [4]. Recent studies confirm that cell and cordless phone microwave can:

- Damage nerves in the scalp

- Cause memory loss and mental confusion

- Cause headaches and induce extreme fatigue

- Create joint pain, muscle spasms and tremors

- Create burning sensation and rash on the skin

- Alter the brain's electrical activity during sleep

- Induce ringing! in the ears, impair sense of smell

- Precipitate cataracts, retina damage and eye cancer

- Open the blood-brain barrier to viruses and toxins

- Reduce the number and efficiency of white blood cells

- Stimulate asthma by producing histamine in mast cells

- Cause digestive problems and raise bad cholesterol levels

- Stress the endocrine system, especially pancreas, thyroid, ovaries, testes

Numerous new therapies hold great promise for the treatment of patients with brain cancer, but the main challenge is to determine which treatment is most likely to benefit an individual patient. DNA-

*Corresponding author: Shalini Gandi, Department of Biochemistry, Andhra University, Visakhapatnam, Andhra Pradesh, India, E-mail: shalini.msc28@ gmail.com

Received September 15, 2011; Accepted December 21, 2011; Published Deceber 23, 2011

Citation: Shalini G, Remya RS, Gayathri G, Vishalakshi V, Phaneendra M (2011) Organ Specific Cancers - Recent Advances in Diagnosis and Treatment. J Cancer Sci Ther S17. doi:10.4172/1948-5956.S17-006

Copyright: ( 2011 Shalini G, et al. This is an open-access article distributed under the terms of the Creative Commons Attribution License, which permits unrestricted use, distribution, and reproduction in any medium, provided the original author and source are credited. 
microarray-based technologies, which allow simultaneous analysis of expression of thousands of genes, have already begun to uncover previously unrecognized patient subsets that differ in their survival. A review says that recent generation of commercially available DNA microarrays are proving to be technically robust and reliable [5].

\section{Head and Neck Cancer}

Head and neck cancers account for $6 \%$ of all cancers worldwide, with nearly 150,000 new cases in Europe alone each year [6]. Head and neck squamous cell carcinoma (HSNCC) is highly invasive, frequently metastasizing to cervical lymph nodes and corresponds with poor prognosis. Elevated Src (sarcoma) kinase activity is linked to the progression of solid tumors, like HNSCC. Src regulates HNSCC proliferation and tumor invasion, with the Src-targeted small molecule inhibitor saracatinib displaying potent anti-invasive effects in preclinical studies [7]. Skin toxicity is the most common adverse event associated with the use of EGFR inhibitors. Radiation dermatitis occurs to some degree in most of the patients who receive radiotherapy, either alone or in combination with EGFR inhibitors. cutaneous toxicity and radiation dermatitis in patients with HNSCC undergoing concurrent treatment with cetuximab and radiotherapy are relative easily manageable and only in very rare instances lead to treatment discontinuation. Lesion monitoring should include weekly visits for minor/moderate lesions and twice per week visits for severe cases [6].

Soft tissue sarcomas of the head and neck are rare mesenchymal malignant neoplasms accounting for less than $10 \%$ of all soft tissue sarcomas and approximately $1 \%$ of all head and neck neoplasms. The cellular features of the fibrohistiocytic tumors sometime make difficult to predict the biological behavior of the soft tissue lesion. In a case study, the patient initially presented with a benign histopathological appearance of the primary lesion later due to its recurrent nature, presented a malignant histopathological picture. Hence a long term, closely monitored post-operative follow up of the benign fibrohistiocytic tumors, is mandatory, especially in recurrences, for the early detection of any possible malignant transformation [8].

Locally advanced head and neck cancer is a great challenge for oncologists. The most aggressive non-surgical treatment is the combination of chemotherapy and radiation however, grade 3 and 4 toxicity also significantly increase along with more intensive schedules. A study was intended to compare concomitant chemoradiation using newer active agent paclitaxel in low dose weekly schedule versus most extensively used agent cisplatin with conventional radiation in locally advanced head and neck cancers. Although no conclusion was drawn as the optimal regimen based on this comparison, concomitant chemoradiotherapy regimens were easily given in the outpatient clinic. The regimen based on paclitaxel was more effective; however, the difference was not enormous because of the shorter duration of follow-up and small sample size. Therefore, further studies are needed with large sample sizes and long duration of follow-up [9]. Laryngeal tumors are among the most cancers in head and neck cancers. One of the common complications of laryngeal surgery is the compromising the patients' voice, so they may not accept the treatment. In the recent years, better techniques of radiotherapy and the use of concurrent chemotherapy have been resulted in survival and local control comparable to surgery especially in early laryngeal lesions. It seems that radiotherapy with or without chemotherapy can be an appropriate alternative to total laryngectomy in preserving of the larynx in different stages of laryngeal cancers [10].

\section{Oral cancer}

Oral squamous cell carcinomas (OSCCs), the most common cancer of the head and neck, accounts for over 300,000 new cancer cases worldwide annually. S- 1 is an oral anticancer drug comprised of tegafur (FT), a prodrug of 5-fluorouracil (5-FU), 5-chloro-2, 4-dihydroxypyridine (CDHP), and potassium oxonate (Oxo). S-1 has been used for adjuvant chemotherapy after primary treatment of head and neck cancer. S-1 monochemotherapy of NAC was a useful and convenient method for outpatients, because it has high effective antitumor activity and low toxicity. Further studies on administration of S-1 NAC to outpatients and confirmation of safety and efficacy may result in increased use [11].

Granulomatous lesions of the Oral and Orophrayngeal submucosal tissues frequently affecting buccal and labial tissues are uncommon and present a diagnostic dilemma because of the wide variety of possible etiologic factors. Cheilitis Granulomatosa (CG) is a rare granulomatous disorder of unknown origin, initially described by German dermatologist Miescher in 1945 as a distinct clinicopathological entity, characterized clinically by diffuse, non tender, soft to firm swelling of one or both lips. The disorder is polyetiologic and may be caused by an alteration in autonomic nervous system function localized to facial skin, resulting in increased vascular permeability and edema. Various treatment modalities such as surgery, drug therapy with antituberculous drugs, antibiotics, vitamins, Phenylbutazone, ACTH, oral and intralesional corticosteroids and boiled water injections have been used in the treatment of CG [12]. Corticosteroids have been shown to be effective in reducing lip swelling and preventing recurrences and are considered the mainstay of therapy. The criteria for choice of treatment would seem to be subjective with little scientific basis for selecting one treatment protocol over another [13]. CG remains an enigmatic disorder with multiple causes. The dental practitioner is likely to encounter patients with this disorder and a proper knowledge of this idiopathic condition is imperative and places a major role in its successful diagnosis and treatment [12].

Although tobacco use and alcohol abuse are the dominant risk factors, human papilloma viruses (HPVs) have been found in a small proportion of OSCCs. Recent studies have suggested an association between periodontal disease and the risk of various human malignant neoplasms including poorly differentiated OSCC. Butyric acid (BA), an extracellular metabolite from periodontopathic bacteria, plays an important role in the progression of periodontal disease. It was concluded that Butyric acid/sodium butyrate increases podoplanin expression and cell migration in certain OSCC cell lines, suggesting that the progression of periodontal disease may promote the progression of OSCC via a podoplanin-dependent pathway [14]. To establish the incidence and risk of developing UAD-SPT in patients diagnosed with OSCC and to appraise the OS rates of patients with SPT in this cohort a study was conducted. The study concludes that UAD-SPT should be clinically divided into the HNSCC-SPT and non-HN subsets for classification purposes. This would have a significant bearing on the perceived overall prognosis of patients [15].

\section{Lung cancer}

Respiration causes tumors in the thoracic and the abdominal cavities to move appreciably in all directions. The range of that motion can be up to $5 \mathrm{~cm}$. The magnitude of the motion and its period are variable from patient to patient, from fraction to fraction and even within each fraction for the same patient. As a result, the management 
of tumor (and organ) motion has posed a major challenge for the radiation therapy community in recent years and is still in the phase of research and development. An investigation quantifying the relation between planning target volumes (PTV) size and its range of motion was presented. Intensity Modulated Radiation Therapy (IMRT) treatment plans were considered, since this technique offers more conformal dose distributions than conventional 3D treatment technique. The presented results outline upper limits for both IMRT and 3D conformal irradiation, i.e. if PTV volume and/or range of motion is greater (for nominal lung volume) than the simulated sizes, then motion management strategy is necessary in order to limit healthy lung toxicity. The readers must be cautioned of the possibility that the tumor size, and its range of motion for a given nominal lung volume, inferred from the figures or the analytic fits to the data may not be realistic if the lesion is in very close proximity to the heart or the spinal cord where those organs at risk OARs might be the dose limiting structures [16].

IMRT is widely accepted as an appropriate method to treat tumors at many different anatomic locations including lung. Dose calculation algorithms that have different degrees of accuracy are used to produce clinical IMRT treatment plans. Monte Carlo (MC) dose calculation was used to evaluate the reliability of plan evaluation parameters compared to a pencil beam (PB) dose calculation for IMRT of the lung. One main conclusion of this work is that the most accurate dose calculation methods (i.e. Monte Carlo) should still be implemented in the clinical routine [17]. One cannot tell a priori which cases will show the largest errors in plan evaluation parameters. Based on the statistical analysis, it was known that there are appreciable differences between the PB and $\mathrm{MC}$ calculations for the targets. Accurate $\mathrm{MC}$ calculations can remove the remaining systematic errors from treatment plans compared to $\mathrm{PB}$ calculations. Lastly, it was concluded that MC algorithms will need to be benchmarked or standardized upon clinical implementation so different levels of accuracy are avoided.

How do researchers define the optimal cores to sample when designing a Tissue microarrays (TMA) study to minimize sampling bias and core artifact? This fundamental question was answered by comparing the staining results from a full-section tissue slide to the virtual TMA and from the actual TMA to the virtual TMA [18]. Virtual and actual TMAs were conducted with three marker antibodies (TTF1, p53, and Ki-67), resulting in six sets of expression staining outcomes to compare with the staining results of the entire tissue slide. TMA results showed that TTF-1 best represented the TVP and was the most consistent across methods. Both virtual and actual TMAs for p53 and Ki-67 tended to underestimate the true value and was most evident for Ki-67 [19]. Results support findings that not all TMA constructs fit one tumor type and researchers need to be aware that different constructs may be needed for different tumor types. Within- and between-tumor heterogeneity could lead to false interpretation of an immunostain; therefore, it was proposed that before using TMAs to analyze thousands of patient samples, a preliminary investigation using virtual TMAs could help discriminate between immunostains that would yield valid results on TMAs and immunostains that would not [18]. Molecular characteristics of Non-small Cell Lung Cancer NSCLC suggests that alkylating agents, such as temozolomide, alone or in combination with MGMT inhibitors, may be viable option to treat selected NSCLC patients. Translational studies provide background molecular data for more rational clinical trial design [20].

To determine the potential role of p53 on the growth inhibition observed with DHA treatment of the human lung adenocarcinomas
A549 (wildtype p53) and H441 (mutant p53, codon 158), p53-specific siRNA's and a chemical inhibitor of p53, pifithrin-a, were employed in vitro. Significant increases in the number of DHA-treated cells by $\mathrm{p} 53$ siRNA or pifithrin-a addition were observed only in the A549 cell lines expressing wildtype $\mathrm{p} 53$, and these correlated with a reduction in the percentage of apoptotic and necrotic cells [21]. This data confirms a role for p53-dependent growth inhibition with DHA treatment. DHA can exhort inhibitory effects independent of p53 status, it also seems apparent that in some cell models there may be a p53- dependent response.

\section{Liver cancer}

Hepatocellular carcinoma (HCC) is the sixth most common cancer in the world, and the third commonest cause of cancer mortality worldwide [22]. Hepatocellular carcinoma (HCC, also called malignant hepatoma) is the most common type of liver cancer (primary hepatic malignancy) in the Asian countries. The prognosis of metastatic hepatocellular carcinoma is very poor if left untreated. A case study of metastatic hepatocellular carcinoma involving the nasal cavity was presented. It revealed the existence of intranasal metastatic HCC, a rare anatomical location for extrahepatic metastasis. The clinicians and the pathologists should always be aware of such unusual presentation owing to the poor prognosis and the possibility of rapid deterioration [23].

Interventional therapy for advanced hepatocellular carcinoma (HCC) is still controversial. A retrospective study was done to evaluate the efficacy of transcatheter arterial infusion (TAI) alone or combined with transcatheter arterial chemoembolization (TACE) on advanced HCC. TAI alone or combined with TACE can improve survival of advanced HCC patients with local advanced or metastatic cancer and play an important role in comprehensive treatment. The portal vein thrombosis and ECOG PS were independent prognostic factors for overall survival (OS). Interventional treatment could greatly prolong the OS of advanced HCC patients [22].

Cancer metastasis requires physiological changes which are regulated by cell signaling molecules. Osteopontin (OPN) is one such molecule secreted phosphoprotein which plays a critical role in metastasis of colon, liver, and breast cancers. OPN binds to it recognized receptors with substantially different affinities, receptor expression varies between cell types, and significant OPN cell surface interactions that are integrin- and CD44-independent. These uncharacterized interactions may reveal important insights into OPN's role in cancer metastasis [24]. The eradication of multiple liver metastases from different tumors is a difficult goal to achieve in most tumors. Study was done to evaluate the response to Y-90 SIR-sphere doses in liver metastases from other primary tumors with particular interest in its effect on metastases from neuroendocrine tumors since they tend to be vascular in nature. One limitation of that study is lack of consistent evaluation of liver function tests or tumor biomarkers at constantly scheduled intervals in many of our patients. It concluded that Y-90 SIRSpheres is a useful therapeutic alternative for multiple liver metastases from a variety of tumors. The side effects are usually well tolerated with mostly grade 1 or 2 toxicity [25].

\section{Breast cancer}

Breast cancer is one of the most frequently diagnosed cancers among women, with an estimated 184,450 new cases and 40,930 deaths in 2008 [26]. It is a steroid hormone-dependent tumor. The importance of hormone status in breast cancer patients is because estrogen and 
progesterone are the key determinants of the therapy. Estrogen (ER) and progesterone (PR) can increase both normal and abnormal breast cell growth. Estrogen and progesterone receptors are highly predictive of breast cancer that will benefit from endocrine therapy. The ER is a nuclear receptor protein that has an estrogen-binding domain and a DNA binding domain. The ER complex binds directly to the DNA and regulates the expression of other genes including the PR. The traditional pathological and morphological examination may be linked to the clinical behavior of tumors, but hormonal status is clearly important as predictive and prognostic factor for therapy. However, the study was limited due to its small cohort of breast cancer patients also there was a drop-outrate of nearly one third of patients during the follow-up courses [27].

Breast cancer incidence and mortality rates decrease with environmental conditions that promote Vitamin D synthesis in human skin including lower latitude and higher personal exposure to sunlight. A future study that takes measurements of Vitamin D status at the end of the winter following varying autumn cloudiness over several years could provide further information about the asserted connection among cloudiness and Vitamin D status, and potential links to breast cancer incidence [28]. The length of breast cancer incidence time series is a limitation of the study. Its strengths lie in the use of independently collected datasets, focus on the autumn season, and on cloudiness as a major modulator of the solar UV reaching the Earth's surface. The proposed mechanism of action through modulation of Vitamin D synthesis in human skin provides a new example of possible interaction between climate variability and human health on decadal scales.

Many viruses have been associated with human breast cancers, including Epstein-Barr and Cytomegalovirus. New evidence has revealed the frequent presence of highrisk human papillomavirus (HPV) strains HPV16 and HPV18 in breast carcinoma biopsies. These findings raise the question of whether HPV may infect developing cancers and mediate their growth and development, as was recently observed with oral cancers. Although HPV is a primary cause of virtually all cervical cancers, it is found as a concomitant infection in many other tumors. While HPV may initiate carcinogenesis in these tumors, recent studies suggest HPV may also modulate the progression or malignancy process in already transformed cancers. Determining the effects of HPV on already transformed breast cancers is an important step towards understanding the factors that will ultimately lead to more accurate prognosis and more effective treatment options for breast cancer patients with concomitant HPV infections [29]. As mentioned earlier osteopontin plays a critical role in metastasis of breast cancer also [24].

Recent data suggests that primary tumour characteristics might provide further important information about predict survival in patients with brain metastases from primary breast cancer. The unique biological features of breast cancer allow for therapeutic approaches that might improve the response of both extra and intra cranial disease maniferstations, e.g., trastuzumab, lapatinib, aromatase inhibitors etc. Lymphopenia, breast cancer subtype and interval from primary tumor diagnosis to development of brain metastases are promising emerging prognostic factors, which have been reported in several retrospective studies. The challenge for the future is the validation of the current preliminary findings on tumor biology and other host factors in a head to head comparision with KPS, extracranial metastases, number of brain metastases, interval and age [30].
Primary breast lymphoma is a rare tumor that presents commonly as a large mass with no specific mammographic appearance and can be confused with poorly differentiated carcinoma on frozen section. Histologically, primary breast lymphoma is predominately of B-cell origin and most commonly large cell type. In addition to physical examination, X-ray of the chest, skull and pelvis is a reliable method for detecting visceral and nodal dissemination and should always be performed. Survival rate of primary breast lymphoma is better as compare to both lobular cases and systemic lymphoma with secondary involvement of breast. Anticancer drugs are main treatment rather than surgery so it is very important to accurately diagnose primary lymphoma of breast [31]

Breast cancers are usually treated with surgery and radiation excretes adverse effects. Azurin, a potent anticancer redox protein secreted by Pseudomonas aeruginosa ( $P$. aeruginosa) species has been reported to have activity against breast cancer cell lines; this had prompted researchers to search for novel methods to enhance this protein's production. Results of a study established that azurin from $P$. aeruginosa strain 2453 may have an extensive antiproliferative effect on breast cancer cells [32]. It is the first report showing that azurin from $P$. aeruginosa strain 2453 induces apoptosis and is mediated through the cell growth arrest resulting in cell cycle analysis at subG1 phase, apoptosis induction via ROS generation, p53 elevation, and up and down regulation of pro and antiapoptotic proteins. The proportion of azurin required for growth inhibition varies according to the cell line and the concentration of azurin used. Future studies should focus on in vivo experiments like intra lesion injection of azurin (from $P$. aeruginosa strain 2453) in animal models of breast cancer; these types of studies will confirm that azurin (from P. aeruginosa strain 2453) can be used as a potential therapeutic agent in patients with breast cancer.

Survivors of breast cancer experience many side effects that influence their quality of life. A study was carried out to assess the quality of life of women with breast cancer at the time of diagnosis [33]. According to that article the quality of life was lowest among the social well-being domain followed by psychological well-being, physical wellbeing, and spiritual wellbeing. In a study [34] reported that almost $90 \%$ of all women with breast cancer reported that they felt depressed, irritable tense or worried [35]. Neutropenic enterocolitis (typhilitis) is a rare complication of chemotherapy-related neutropenia in breast cancer patients. A case study was done to those patients, which concludes that attention should be paid even to vague abdominal discomfort. Urgent CT scan evaluation and early surgery appear to be of great importance [36].

\section{Prostate cancer}

Prostate cancer is the most prevalent and commonly diagnosed male cancer. Approximately 217,730 new cases of prostate cancer and 32,050 deaths are projected to occur in USA in 2010. Though radiotherapy is a common treatment for prostate cancer failure is observed 30 to $40 \%$ of the time. The effect of curcumin and radiation on cell viability, apoptosis and clonogenic cell death was examined in LNCaP (wild type p53) and PC3 (mutant p53) prostate cancer cells. The examination concludes that curcumin appears to radiosensitize prostate cancer cells and may be a possible adjuvant to radiotherapy in the treatment of prostate cancers [37]. The causes increasing trend of prostate cancer incidence are not completely known. Emerging evidences suggest that among many risk factors, inflammation is the major risk factor for developing prostate cancer. It was proposed that exposure to environmental factors such as infectious agents, dietary agents and saturated lipids leads to injury of 
the prostate due to chronic inflammation and regenerative risk factor lesions referred to as proliferative inflammatory atrophy [38].

Now a day's treatment of prostate cancer is taking new pathways from prostatectomy and radiation therapy, which may often be supplemented with hormonal therapies. In prostatectomy and radiation therapy recurrence is common, and many develop metastatic prostate cancer for which chemotherapy is only moderately effective. Hence novel therapies are needed for the treatment of the malignant forms of prostate cancer, such as hormone refractory (HRPC) or castration resistant prostate cancer (CRPC) [39]. A study reveals that two related drugs, finasteride and dutasteride, reduce the incidence of prostate cancer and the risk of diagnosis [40]. A study was conducted to determine whether there is any relation between the histologic grade, tumour marker level, and the aggressiveness of the tumour itself in ethnic group. It also correlated the Gleason score with prostate specific antigen (PSA) level and probability of positive bone scintigraphy results. Progressive rising incidence of bone metastasis on radionuclide bone scan in relation to the PSA levels in patients newly diagnosed of prostate cancer was proven [41]. Using PSA as an indicator for the presence of bony metastases rather than routine bone scans would have large economic savings given the population size.

Multicellular spheroid (MCS) culture models have become a mainstream culture model for tumor biology and identification of anticancer resistance mechanisms that poorly reflect the structural characteristics seen in vivo. They better mimic the growth characteristics of in vivo solid tumors. MCS culture models are a good model for understanding the mechanisms of resistance to chemotherapy, radiotherapy, and androgen ablation, and discovery of new targets for prostate cancer, especially androgen-independent cancer. The characteristics of prostate cancer MCS (p27 and PARP expression, and epigenetics), and underlined the tumor microenvironment as target for prostate cancer therapy has been highlighted by a review [42]. MCS culture models appear to contribute to the identification of cancer stem cells from the prostate. Further studies are necessary to discover new targets for prostate cancer.

\section{Pancreatic, gastric and colorectal carcinoma}

Pancreatic cancer remains a major unsolved health problem. The main risk factors of pancreatic cancer are smoking, age, and some genetic disorders, although the primary causes are poorly understood [43]. The efficacy of combined radiation and fluorouracil as adjuvant therapy for pancreatic cancer was suggested by a prospective randomized study conducted by the Gastrointestinal Tumor Study Group (GITSG) [44]. The risk of pancreatic cancer is significantly elevated in patients with chronic pancreatitis and appears to be independent of sex, country, and type of pancreatitis [45].

Gastric signet ring cell carcinoma (SRC) is poorly distinguished adenocarcinoma in which the tumour cells invade singly or in small groups. It occurs more frequently among women and young patients. A regulated course of Epirubicin, Oxaliplatine and Capecitabine (EOX) remains the standard treatment of advanced Primary gastric signet-ring cell carcinoma. A complete pathologic response to EOX was given by a case study [46]. Non-Hodgkin's Lymphoma of the stomach is the most common group of primary extranodal lymphomas. Microsatellite insatiability (MSI) plays an important role in development of primary gastric lymphoma mainly in DLBCL types. Role of Helicobacter pylori eradication in controlling primary gastric lymphoma and its effect in correction of MSI was confirmed by Azza Eldin et al. [47].
Gastric cancer is the 2rd most common tumor in the world. Incidence rates in men are twice those in women, in both low-risk and high-risk areas [48]. Overall, it still remains among the leading killer cancers representing 3\% of all cancer deaths [49]. Mutation pattern of TP53 revealed certain peculiarities in having maximum mutations in exon-5, high frequency of deletions and insertions besides no mutation at hotspot codons. A study, suggests TP53 as a potential molecular marker and prognostic tool [48]. But these observations need further investigations in a bigger cross section of the gastric cancer patients.

Colon, or colorectal, cancer is cancer that starts in the large intestine (colon) or the rectum (end of the colon). A superficial orthotopic implantation model of colon cancer was established by pulling out the cecum and suturing it to the peritoneum. It is a valuable orthotopic implantation model for evaluating colon cancer [50]. Since the 1970s it was considered that bile acids are promoters of colon cancer, but lack carcinogenic activity. But recent evidences indicate that bile acids indeed, act as carcinogens. Bile acid deoxycholic acid (DOC) is a carcinogen, not merely a promoter. Further results of a study suggest that, in humans, the association of colon cancer with a high fat diet is mediated by persistent exposure to high physiologic levels of bile acids, especially DOC [51].

\section{Renal cancer}

Squamous cell carcinoma of the renal pelvis and ureter is a rare malignancy. Nephrectomy with ureterectomy is the treatment of choice in these patients. There is lack of evidence regarding survival benefit with chemo-radiation following surgery but it is still advocated by some with the hope of increasing survival. A study says that biopsy from the renal pelvis or calyceal wall should be considered during the treatment of stone disease, in patients having long-standing history of large renal calculi or staghorn calculus. Such patients are susceptible of harboring occult or overt malignancy along with the renal stone disease [52].

A study assessed toxicity and potential efficacy of low-dose granulocytemacrophage colony-stimulating factor (GM-CSF), interferon alpha (IFN) and interleukin 2 (IL-2) postoperatively in patients with high-risk renal cell carcinoma (RCC) [53]. Drug-induced hepatotoxicity is an infrequent but life-threatening complication. Sunitinib is a multitargeted receptor tyrosine kinase inhibitor approved for treatment of advanced renal cell carcinoma and gastrointestinal stromal tumor. Although the targeted agents used in the treatment of RCC are reasonably well tolerated, their toxicity on a long term basis is unknown. Clinicians should be aware of this possible adverse effect of sunitinib, and continued pharmacovigilance is imperative to accurately quantify the possible risk of sunitinib-related hepatotoxicity [54].

\section{Leukemia}

Leukemia is an unregulated proliferation of haematopoietic tissue that progressively displaces normal blood cell elements. A study included Horseradish peroxidase conjugated Canavalia gladiata lectin used to evaluate its binding property to different types of leukemic cells and to investigate whether the lectin could be used for the typing of leukemias. The presence of lectin receptors on the blastic cells could be of considerable importance because they may serve as targets for drug binding and enable selective destruction of pathogenic cells [55]. Lectin cytochemical studies in leukemia revealed its usefulness in differentiating lymphoid leukemias from myeloid leukemias. Advances in the design and production of immunotoxins containing the bacterial protein toxin $\mathrm{PE}$ have made possible the clinical testing of promising new targeted agents for treatment of hematologic malignancies [56]. 
A milestone in the treatment of chronic lymphocytic leukemia (CLL) was reached with the incorporation of immunotherapy with conventional chemotherapy. The fludarabine/cyclophosphamide/ rituximab combination has demonstrated survival advantage for the first time in the treatment of CLL [57]. Development of specific targetbased drugs for Juvenile myelomonocytic leukemia (JMML) remains a big challenge and represents a promising direction in this field. Scientists and clinicians should continue to study molecular defects in JMML in a concerted effort to define novel therapeutic targets and to develop effective, less toxic, therapeutic interventions [58].

A case report highlights some of the histopathological difficulties in the diagnosis of primary bronchial Sialadenoma papilliferum, a rare tumor. Owing to its overall rarity, the long-term biologic behavior of bronchial Sialadenoma papilliferum still remains unknown [59].

\section{Bone Cancer}

Osteosarcoma is a bone tumor and can occur in any bone, usually in the extremities of long bones near metaphyseal growth plates. Pain in the involved region of bone is a unique clinical presentation of osteosarcoma, which is unusual of other tumors and thus osteosarcoma can be confused with other inflammatory lesions. Radiographic evaluation plays an important role in the initial diagnosis of osteosarcoma. In addition, CT scans are excellent for demonstrating the degree of intramedullary extension, cortical involvement and soft tissue involvement. A case study says that the treatment and prognosis for osteosarcoma depend to a large extent on early diagnosis and radical surgery [60].

Prostate cancers often metastasize to bone. It is hypothesized that interactions between osteoblasts and cancer cells lead to establishment of prostate cancer skeletal metastasis. Interactions between prostate cancer cells and mature bone-producing osteoblasts resulted in gene expressional changes that may increase cells' ability to establish bone metastasis. The potential for inducing expressional changes seems to be crucial dependent of the differentiation status of osteoblasts. Because of the complexity of the metastasis process, it is a huge challenge to elucidate the interplay between prostate carcinoma cells and their favourite homing site in the skeletal system. A study says that interactions between prostate cancer cells and mature bone-producing osteoblasts might be potential targets when developing new cancer therapy strategies [61].

\section{Cervical cancer}

Management of locally advanced cervical cancer is a challenge to oncologists [62]. Cervical cancer is a malignant neoplasm arising from cells originating in the cervix uteri. One of the most common symptoms of cervical cancer is abnormal vaginal bleeding, but in some cases there may be no obvious symptoms until the cancer has progressed to an advanced stage. The mainstay of treatment in cancer cervix is radiotherapy. The hormonal treatment enabled control of most climacteric symptoms, without any serious side-effects as well relieved rectal, bladder, and vaginal postradiological complications [63]. A study on continuous hyperfractionated accelerated radiotherapy (CHART) in locally advanced cervical cancer observed that 10-days CHART technique is a safe and well tolerated regimen treatment which resulted in optimal local control rate and toxicity profile [62].

\section{Ovarian cancer}

Ovarian cancer is a cancerous growth arising from the ovary. Symptoms may include bloating, pelvic pain, difficulty eating and frequent urination, and are easily confused with other illnesses [64]. Primary ovarian carcinoid tumors are very rare. Surgical excision is recommended for cancers at early stages, while management in advanced forms is rather doubtful. In some cases, radical surgery remains the best treatment in terms of survival and palliation [65]. A case study presents a rare case of ovarian mixed, trabecular and insular, carcinoid with lymph node metastases [66]. It says that there was no evidence on the benefits of adjuvant therapy or the type of drug; the patient was therefore referred to in the standard chemotherapy regimen for epithelial ovarian cancer. It appeals that further studies and close follow-up for the correct outcome evaluation of the patient are recommended.

Evaluation of efficacy and toxicity of radiation therapy for recurrent epithelial ovarian cancer after chemotherapy according to the disease status was done. The study showed that RT is a treatment to be considered for recurrent EOC, especially in good responders to platinum or patients with solitary relapsed lesion, even though we were not able to analyze the dose-response relationship and the effect of RT patterns [67].

\section{Anal cancer}

Anal cancer is a type of cancer arising from the anus, the distal orifice of the gastrointestinal tract. Despite the small number of patients affected by anal cancer, it remains as a most challenging cancer to treat. Centralized treatment of anal canal neoplasms would allow the elaboration of clinical trials to determine the adequate treatment (chemotherapy and radiotherapy), and also the establishment of guidelines for monitoring the patients [68]. A report was made on preliminary results of treatment of anal carcinoma by radiotherapy and chemotherapy. It suggests that a greater number of patients and a longer follow up period is required to establish radiotherapy and chemotherapy as a valid alternative to abdomino-perineal surgery for the treatment of anal carcinoma [69].

Till now it was thought that Kaposi's sarcoma and non-Hodgkin lymphoma are the only cancers that have been strongly associated with AIDS. But a research study establishes a strikingly increased risk of anal cancer among people with AIDS. Clinicians should be aware that AIDS patients have an increased risk of anal cancer [70]. A case study says that early treatment strategies in this type of patients group employed reduced dosages of chemotherapy or radiotherapy alone to reduce toxicity. It also suggests that radical chemoradiation may be given safely at conventional doses in HIV-positive patients, with a high complete response rate [71].

\section{Advancement in cancer therapies}

Recently a research conveys that inhibitors of Tyrosine Kinases (TKI) and Small Interfering RNAs (siRNA) are promising targeted cancer treatments [72]. Development of inhibitors of TKI and siRNA, with effects better than or at least similar to conventional therapy must be given high priority. Tolerable side effects are also critical to successful implementation. Only through such efforts may such treatment be fully developed and find its place in the treatment of malignant tumours, solely, as primary choice, or in combination with conventional cancer therapy. RNA interference is likely to become a treatment option for some cancers, either alone or as a supplement to traditional treatment [72].

Antioxidants are considered as one of the effective solutions for the oxidative stress. Now a days they are used in the prevention and 
Citation: Shalini G, Remya RS, Gayathri G, Vishalakshi V, Phaneendra M (2011) Organ Specific Cancers - Recent Advances in Diagnosis and Treatment. J Cancer Sci Ther S17. doi:10.4172/1948-5956.S17-006

treatment of numerous diseases including inflammatory and infectious diseases, pre and post cancer therapy. Potent antioxidant activities were observed from dendritic antioxidant (zero generation -G0) derived from syringaldehyde. Its analogs were derived from vanillin and 5-iodovanillin. A study presented the characterization and molecular interaction of the dendritic antioxidants with $p B R 322$ plasmid DNA using computer simulations [73]. Based on interaction and binding energy the study concluded that syringaldehyde-derivatized G0 dendrimer was the most favorable entity for pBR322 DNA, followed by quercetin.

\section{Conclusion}

This review covers different Organ Specific Cancers and recent advantages in their diagnosis and treatment. These recent advances in diagnosis and treatment of cancer have led to an increase in cancer survival and hence, there is a greater emphasis on quality beside quantity of survival. Further investigation on the quality oriented cancer therapies leads to quality oriented lives in future.

\section{References}

1. Sudhakar A (2009) Oral Submucous Fibrosis: Review on Etiopathogenesis. J Canc Sci Ther 1: i-iv.

2. Park J, Kim J, Yoo E, Lee H, Chang JH, et al. (2011) Detection of Smal Metastatic Brain Tumors: Comparison of 3D Contrast-Enhanced Whole-Brain Black-Blood Imaging and MP-RAGE Imaging. Invest Radiol [Epub ahead of print].

3. Elena IF, Eric CH (2005) Stem cells and brain cancer. Experimental Cell Research 306: 323-329.

4. Hardell L, Hallquist A, Mild KH, Carlberg M, Påhlson A, et al. (2002) Cellular and cordless telephones and the risk for brain tumours. Eur $\mathrm{J}$ Cancer Prev 11: 377-386.

5. Paul SM, Timothy FC, Stanley FN (2004) DNA-microarray analysis of brain cancer: molecular classification for therapy. Nat Rev Neurosci 5: 782-792.

6. Ammer AG, Kelley LC, Hayes KE, Evans JV, Lopez-Skinner LA, et al. (2009) Saracatinib Impairs Head and Neck Squamous Cell Carcinoma Invasion by Disrupting Invadopodia Function. J Cancer Sci Ther 1: 052-061.

7. Mesía R, Vilajosana E, Lozano A, Es ${ }^{T M}$ teller L, Silvia V (2009) Management of Cutaneous Toxicity and Radiation Dermatitis in Patients with Squamous Cancer of the Head and Neck Undergoing Concurrent Treatment with Cetuximab and Radiotherapy. J Cancer Sci Ther 1: 028-033.

8. Bali A, Singh MP, Padmavathi, Khorate M, Ahmed J (2010) Malignant Fibrous Histiocytoma - An Unusual Transformation from Benign to Malignant. J Cancer Sci Ther 2: 053-057.

9. Hamed RH, Elzahaf E (2011) Low Dose Weekly Paclitaxel Versus Low Dose Weekly Cisplatin with Concomitant Radiation in Locally Advanced Head and Neck Cancers. J Cancer Sci Ther 3: 166-171.

10. Shaghayegh K, Mahdi A, Ali K (2010) Larynx Preserving Treatments in the Early and Advanced Laryngeal Cancers; A Retrospective Analysis. J Cancer Sci Ther 1: 008-010.

11. Yokoe H, Kasamatsu A, Ogawara K, Ishigami T, Sato $Y$, et al. (2010) Neoadjuvant Chemotherapy with S-1 for Patients with Oral Squamous Cell Carcinoma. J Cancer Sci Ther 2: 132-135.

12. Goel S, Khorate M, Nahar P, Ahmed J (2010) Cheilitis Granulomatosa - An Uncommon Clinicopathological Entity: A Case Report. J Cancer Sci Ther 2: 091-093.

13. Grave B, McCullough M, Wiesenfeld D (2009) Orofacial granulomatosis--a 20year reviews. Oral Dis 15: 46-51.

14. Miyazaki Y, Kikuchi K, González-Alva P, Inoue H, Noguchi Y, et al. (2010) Association of Butyric Acid Produced by Periodontopathic Bacteria with Progression of Oral Cancer. J Cancer Sci Ther 2: 026-032.

15. Farhadieh RD, Otahal P, Taghavi K, Salardini A, Russell P, Smee R (2011) Second Primary Tumours of the Head and Neck are not Associated with
Adverse Overall Survival in Oral Squamous Cell Carcinomas. J Cancer Sci Ther 3: 030-034.

16. Mihaylov IB, Lerma FA, Moros EG (2010) Relation Between Tumor Size and Range of Motion in IMRT Treatment Planning for Thoracic Lesions. J Cance Sci Ther 2: 100-104

17. Aljarrah K, Pawlicki T, Niemierko A, Mell LK, Jiang SB (2010) A Clinical Study of MLC-Based IMRT Lung Dose Calculation Accuracy on Plan Evaluation Parameters. J Cancer Sci Ther 2: 074-081.

18. Wampfler JA, Aubry MC, Yang P, Riehle DL, Savci-Heijink CD (2011) Determining the Optimal Numbers of Cores Based on Tissue Microarray Antibody Assessment in Non-Small Cell Lung Cancer. J Cancer Sci Ther 3 120-124.

19. Macdonald C, Michael A, Colston K, Mansi J (2004) Heterogeneity of immunostaining for tumour markers in non-small cell lung carcinoma. Eur $J$ Cancer 40: 461-466.

20. Emery IF, Battelli C, Cai L, Hayes DM (2011) Assessment of DNA Repair Mechanisms to Determine the Susceptibility of Non-small Cell Lung Cancer to Alkylating Agents. J Cancer Sci Ther 3: 092-095.

21. Kikawa KD, Noah T, Ahwah SM, Pardini RS (2011) Docosahexaenoic Acid (DHA) Induces P53-Dependent Growth Inhibition in Transformed Colon and Lung Cell Lines Expressing Wildtype P53. J Cancer Sci Ther 3: 182-185.

22. Hao M, Lin H, Chen Q, Yu W, Zhou D, et al. (2011) Efficacy of Transcatheter Arterial Infusion Alone or Combined with Transcatheter Arteria Chemoembolization on Advanced Hepatocellular Carcinoma. J Cancer Sci Ther 3: 130-133.

23. Liu CY, Chang LC, Yang SW (2011) Metastatic Hepatocellular Carcinoma to the Nasal Cavity: A Case Report and Review of the Literature. J Cancer Sc Ther 3: 081-083.

24. Mi Z, Guo H, Markovic J, Kuo PC (2009) Characterization of Osteopontin Binding Kinetics In MDA-MB231 Breast and SK-Hep-1 Liver Cancer Cells. J Cancer Sci Ther 1: 047-051.

25. Gayed IW, Wahba H, Wan D, Joseph U, Murthy R (2010) Effect of Y-90 SIRSpheres Therapy for Multiple Liver Metastases in a Variety of Tumors. J Cancer Sci Ther 2: 043-046.

26. Bareggi Renato NV, Paola N (2010) New Targeted Therapies Against Breast Cancer. J Carcinogene Mutagene 1:110.

27. Jamil K, Kumar K, Fatima SH, Rabbani S, Kumar R, et al. (2009) Clinica Studies on Hormonal Status in Breast Cancer and its Impact on Quality of Life (QOL). J Cancer Sci Ther 1: 083-089.

28. Stajner I (2009) Cloudiness and Breast Cancer. J Cancer Sci Ther 1: 034-040.

29. Kingsley K, Zuckerman J, Davis M, Matteucci M, Knavel A, et al. (2009) Induction of Differential Growth in vitro by High-risk Human Papillomavirus in Human Breast Cancer Cell Lines is Associated with Caspase Dysregulation. J Cancer Sci Ther 1: 062-071.

30. Nieder C, Spanne O, Bilberg I, Dalhaug A (2010) Primary Tumour Characteristics as Potential Prognostic Factors in Brain Metastases from Breast Cancer. J Cancer Sci Ther 2: 070-073.

31. Nigam A, Singh AK, Singh SK, Singh N, Singh N, et al. (2011) Primary Mammary (Non-Hodgkin) Lymphoma of Breast: A Case Report. J Cancer Sci Ther 3: 173-175.

32. Ramachandran S, Sarkar S, Mazumadar A, Mandal M (2011) Azurin Synthesis from Pseudomonas Aeruginosa MTCC 2453, Properties, Induction of Reactive Oxygen Species, and p53 Stimulated Apoptosis in Breast Carcinoma Cells. J Cancer Sci Ther 3: 104-111.

33. Pakseresht S, Ingle GK, Garg S (2011) Quality of Life of Women with Breas Cancer at the Time of Diagnosis in New Delhi. J Cancer Sci Ther 3: 066-069.

34. Ferrell BR, Grant M, Funk Bet, Otis-Green S, Garcia N (1998) Quality of life in breast cancer. Part II: Psychological and spiritual well-being. Cancer Nursing 21: 1-9.

35. Roth RS, Lowery JC, Davis J, Wilkins EG (2005) Quality of life and affective distress in women seeking immediate versus delayed breast reconstruction after mastectomy for breast cancer. Plast Reconstr Surg 116: 993-1002. 
Citation: Shalini G, Remya RS, Gayathri G, Vishalakshi V, Phaneendra M (2011) Organ Specific Cancers - Recent Advances in Diagnosis and Treatment. J Cancer Sci Ther S17. doi:10.4172/1948-5956.S17-006

36. Lombardi D, Venturini S, Veronesi A (2011) Neutropenic Enterocolitis as Possible Complication of Docetaxel and Epirubicin Chemotherapy for Breast Cancer: Report of 3 Cases. J Cancer Sci Ther 3: 186-187.

37. Nayak BK, Krishnegowda NK, Galindo CA, Meltz ML, Swanson GP (2010) Synergistic Effect Between Curcumin (diferuloylmethane) and Radiation on Clonogenic Cell Death Independent of p53 in Prostate Cancer Cells. J Cancer Sci Ther 2: 171-181.

38. Singh RK, Sudhakar A, Lokeshwar BL (2010) Role of Chemokines and Chemokine Receptors in Prostate Cancer Development and Progression. J Cancer Sci Ther 2: 094-099.

39. Fu W, Madan E, Yee M, Zhang H (2011) Progress of molecular targeted therapies for prostate cancers. Biochim Biophys Acta [Epub ahead of print].

40. Justman S (2011) What's Wrong With Chemoprevention of Prostate Cancer? Am J Bioeth 11: 21-25.

41. Abdellatif J, Hajji F, Elonodo JC, Ghadouane M, Ameur A, et al. (2011) Update the Indicator Role of Serum PSA Level and Gleason Score of the Biopsy for the Presence of Bony Metastases: Bone Scan Findings in a North African Ethnic Group. J Cancer Sci Ther 3: 112-115.

42. Kurioka D, Takagi A, Yoneda M, Hirokawa Y, Shiraishi T, et al. (2011) Multicellular Spheroid Culture Models: Applications in prostate Cancer Research and Therapeutics. J Cancer Sci Ther 3: 060-065.

43. Donghui Li , Keping Xie , Robert Wolff , James L Abbruzzese (2004) Pancreatic cancer. The Lancet 363: 1049-1057.

44. Martin H. Kaiser, Susan S. Ellenberg (1985) Pancreatic Cancer: Adjuvan Combined Radiation and Chemotherapy Following Curative Resection. Arch Surg 120: 899-903

45. Albert BL, Patrick M, Giorgio C, Rudolf WA, Paul GL, et al. (1993) Pancreatitis and the Risk of Pancreatic Cancer. N Engl J Med 328: 1433-1437.

46. Akasbi Y, Arifi S, Ousadden A, Tizniti S, Amarti A, et al. (2011) Complete Pathologic Response in Advanced Primary Gastric Signet-Ring Cell Carcinoma: A Case Report. J Cancer Sci Ther 3: 076-078.

47. Ezz Eldin AM, Alkareemy ER, Zedan A, Abdel Warith A, Zaky AH, et al. (2010) The Role of Anti-Helicobacter Pylori Therapy in Remission Induction of Primary Gastric Lymphoma by Analysis of Microsatellite Instability. J Carcinogene Mutagene 1:107.

48. Abdullah S, Sameer S A, Dil-Afroze, Syeed N, Das BC, et al. (2010) P53- The Molecular Guardian Crashes in Gastric Adenocarcinomas - A Study in an Ethnic Kashmiri Population. J Carcinogene Mutagene 1:106.

49. Ekstrom AM, Hansson LE, Signorello LB, Lindgren A, Bergstrom R, et al. (2000) Decreasing incidence of both major histologic subtypes of gastric adenocarcinoma-a population-based study in Sweden. Br J Cancer 83: 391 396.

50. Jin H, Yang Z, Wang J, Zhang S, Sun Y, et al. (2010) A Superficial Colon Tumor Model Involving Subcutaneous Colon Translocation and Orthotopic Transplantation of Green Fluorescent Protein-Expressing Human Colon Tumor. J Carcinogene Mutagene 1:103.

51. Bernstein C, Payne CM, Bernstein H (2011) Bile Acids: Promoters or Carcinogens in Colon Cancer? J Carcinogene Mutagene 2:101e.

52. Singh V, Sinha RJ, Sankhwar SN, Mehrotra B, Ahmed N, et al. (2010) Squamous Cell Carcinoma of the Kidney - Rarity Redefined: Case Series with Review of Literature. J Cancer Sci Ther 2: 087-090.

53. Tsimafeyeu I, Demidov L, Kharkevich G, Petenko N, Volkova M, et al. (2010) Granulocyte-Macrophage Colony-Stimulating Factor, Interferon Alpha and Interleukin-2 as Adjuvant Treatment for High-Risk Renal Cell Carcinoma. J Cancer Sci Ther 2: 157-159.

54. El Mesbahi O, El M'rabet FZ (2011) Reversible Hepatic Cytolysis Secondary to Sunitinib in Metastatic Renal Carcinoma. J Cancer Sci Ther 3: 047- 049.

55. Nair LS, Mahesh S, Smitha LS, Sujathan K, Remani P (2011) Expression of Canavalia gladiata lectin in leukemic cells. J Cancer Sci Ther 3: 088-091.

56. Lechleider R, Pastan I (2011) Advances in the Development of Anti-CD22 Immunotoxins Containing Pseudomonas Exotoxin for Treatment of Hematologic Malignancies. J Cancer Sci Ther 3: 050-052.
57. Aisha M, Taimur S, Aneel P, Kena CM, Kasyapa SC, et al. (2011) Targeted treatment for chronic lymphocytic leukemia. Onco Targets Ther 4: 169-183.

58. Liu X, Sabnis H, Bunting KD, Qu CK (2012) Molecular targets for the treatment of juvenile myelomonocytic leukemia. Adv Hematol 2012:308252.

59. Falkenstern Ge RF, Ott G, Friedel G, Markmann HU, Kohlhäufl M, Kalla $J$ (2011) Bronchial Sialadenoma Papilliferum: A Very Rare Cause of Hemoptysis. J Cancer Sci Ther 3: 020-021.

60. Khorate MM, Goel S, Singh MP, Ahmed J (2010) Osteosarcoma of Mandible: A Case Report and Review of Literature. J Cancer Sci Ther 2: 122-125.

61. Skogseth H, Dybwad M, Flatberg A, Halgunset J (2010) Mature BoneProducing Osteoblasts Alter Gene Expression of Metastasis Related Genes in Prostate Cancer Cells. J Carcinogene Mutagene 1:111.

62. Biswal BM, Ahmad NM, Hanafia ZA, Zakaria A, Othman NH, et al. (2011) Pilo Study on Continuous Hyperfractionated Accelerated Radiotherapy (CHART) and High Dose Rate Brachytherapy in Locally Advanced Cervical Cancer. $J$ Cancer Sci Ther 3: 125-129.

63. Elzbieta $P$ (1987) Hormonal replacement therapy in patients after cervical cancer treatment. J Gynecol Oncol 26: 169-177.

64. Laura J (2010) Test to Help Determine If Ovarian Masses Are Cancer. Wall Str J.

65. Athavale RD, Davies-Humphreys JD, Cruickshank DJ (2004) Primary carcinoid tumours of the ovary. J Obstet Gynaecol 24: 99-101.

66. Cafà EV, Angioli R, Scollo P (2010) Ovarian Carcinoid Tumor with Noda Metastases: Case Report. J Cancer Sci Ther 2: 120-121.

67. Lee SW, Park SM, Kim YM, Kim YS, Choi EK, et al. (2011) Radiation therapy is a treatment to be considered for recurrent epithelial ovarian cancer after chemotherapy. Tumori 97: 590-595.

68. Menéndez $P$, Rabadán L, Villarejo $P$, Padilla $D$, Pardo $R$ (2011) Is the Centralized Treatment of Small Cell Carcinoma of Anal Canal Necessary? A Case Report. J Cancer Sci Ther 3: 158-160.

69. San MS, Armando S, Arraztoa J, Morales Y (1989) Anal neoplasms. Treatment with radiotherapy and exclusive chemotherapy. Rev Med Chil 117: 1137-1140.

70. Melbye M, Coté TR, Kessler L, Gail M, Biggar RJ (1994) High incidence of anal cancer among AIDS patients. The AIDS/Cancer Working Group. Lancet 343 636-639.

71. Cleator S, Fife K, Nelson M, Gazzard B, Phillips R, et al. (2000) Treatment of HIV-associated invasive anal cancer with combined chemoradiation. Eur $J$ Cancer 36: 754-758.

72. Skogseth H, Tvedt KE, Halgunset J (2011) Inhibitors of Tyrosine Kinases (TKI) and Small Interfering RNAs (siRNA) are Promising Targeted Cancer Treatments. J Carcinogene Mutagene 2:122.

73. Chhetri P, Lee C, Rakesh L (2011) Molecular Modeling Studies of Interaction Between Plasmid DNA (pBR322) and Dendritic Antioxidants. J Cancer Sci Ther 3: $220-227$. 